\title{
Supplement for "Diffusion of Organic Molecules as a Function of Temperature in a Sucrose Matrix (a Proxy for Secondary Organic Aerosol)",
}

Kristian J. Kiland, Adrian M. Maclean, Saeid Kamal, and Allan K. Bertram Department of Chemistry, The University of British Columbia, Vancouver, BC, V6T 1Z1, Canada

Correspondence to: Allan K. Bertram (bertram@chem.ubc.ca)

\section{S1. Preparation of thin films containing sucrose, water, and rhodamine-6G}

Rectangular area fluorescence recovery after photobleaching (rFRAP) was used to measure diffusion coefficients of rhodamine-6G (R6G) within sucrose-water solutions. For these measurements, thin films containing sucrose, water, and trace amounts of R6G were required. To prepare these thin films, first a solution containing $20 \% \mathrm{wt} / \mathrm{wt}$ sucrose-water and $0.04 \mathrm{mM}$ R6G was prepared gravimetrically. Droplets of this solution were then deposited on clean and dry hydrophobic siliconized glass slides (Hampton Research) using a needle. Droplets ranged in diameter from approximately $200 \mu \mathrm{m}$ to $1 \mathrm{~mm}$. To set the water activity $\left(a_{w}\right)$ within the droplets, the glass slides holding the droplets were placed within sealed glass jars containing a saturated salt solution with a known relative humidity (RH). At equilibrium, $a_{w}$ within the droplets equals $\mathrm{RH} / 100$. The time needed for droplets to reach equilibrium with the surrounding $\mathrm{RH}$ was estimated using the following equation: ${ }^{1}$

$$
\tau_{\text {diff,H2O }}=\frac{d_{p}^{2}}{4 \pi^{2} D_{H_{2} O}}
$$


Where $\tau_{\text {diff,H2O }}$ is the characteristic mixing time of water within the droplets due to molecular diffusion, $d_{p}$ is the diameter of the droplet, and $D_{H 2 O}$ is the diffusion coefficient of water in a sucrose-water mixture. ${ }^{2}$ The characteristic mixing time refers to the time after which that the concentration of the water at the center of the droplet has less than a $1 / e$ deviation from the

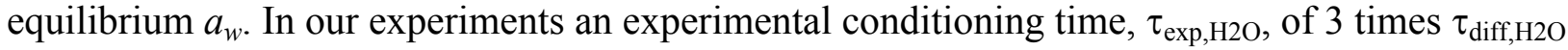
was used to ensure near equilibrium between the droplets and the surrounding RH. The $\tau_{\exp , \mathrm{H} 2 \mathrm{O}}$ value ranged from approximately 3 minutes for $a_{w}=0.85$ to about a week for $a_{w}=0.43$. Table S1 lists the saturated salts used to condition samples to each $\mathrm{RH},{ }^{3}$ and also shows the experimental conditioning time for a $400 \mu \mathrm{m}$ droplet (typical size) over each salt.

Table S1. Saturated salt solutions used to achieve fixed $a_{w}$ of samples and corresponding experimental conditioning time, $\tau_{\text {exp,H2O }}$, for $400 \mu \mathrm{m}$ droplet (typical diameter in our experiments).

\begin{tabular}{|c|c|c|}
\hline Water activity (at 293 K) & Saturated salt $^{3}$ & $\begin{array}{c}\tau_{\text {exp,H2O for a } d_{p}=400} \\
\mu \mathrm{m} \text { droplet }\end{array}$ \\
\hline 0.43 & $\mathrm{~K}_{2} \mathrm{CO}_{3}$ & $6.7 \mathrm{~d}$ \\
\hline 0.59 & $\mathrm{NaBr}$ & $6.2 \mathrm{hr}$ \\
\hline 0.75 & $\mathrm{NaCl}$ & $22 \mathrm{~min}$ \\
\hline 0.85 & $\mathrm{KCl}$ & $3 \mathrm{~min}$ \\
\hline
\end{tabular}

Following conditioning, the glass jar containing the droplets was brought inside a sealed glove bag. RH inside the glove bag was controlled using a humidified flow of nitrogen gas and monitored using an Omega RH85 handheld hygrometer $( \pm 2.5 \%)$. When the RH inside the bag reached the same RH as inside the jar, the jar was opened, and a second hydrophobic siliconized glass slide was placed on top of the glass slide holding the droplets, sandwiching the droplets and producing a thin film with a thickness approximately 35 to $80 \mu \mathrm{m}$. An approximate minimum thickness $(35-50 \mu \mathrm{m})$ of the thin films was set by adding aluminum spacers between the two 
slides. Vacuum grease was applied to the perimeter of the first glass slide, to seal the thin film from the surrounding environment. A schematic of the thin film samples is shown in Figure S1.

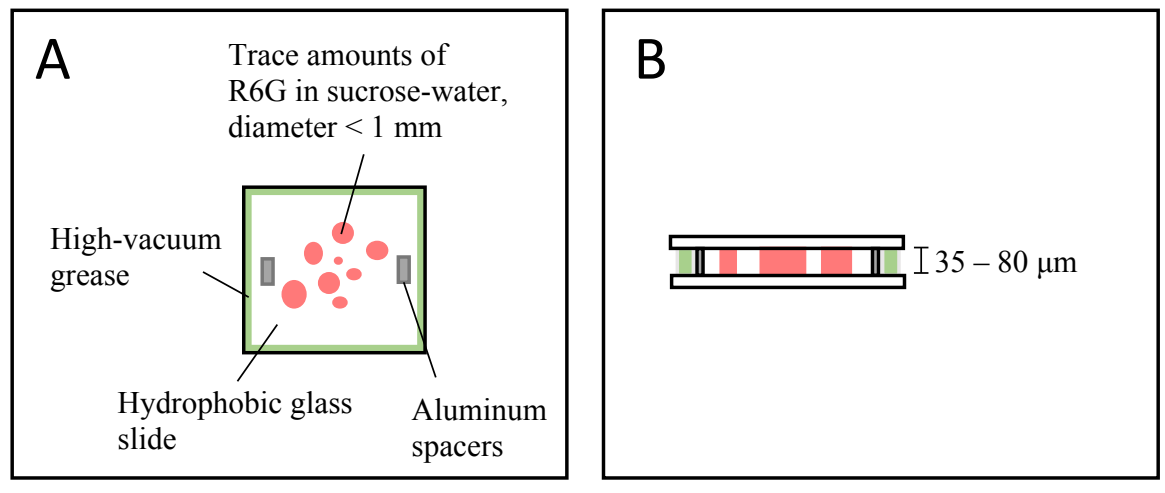

Figure S1. Schematic of thin film samples used in the rFRAP experiments. Panel A shows a top view of the prepared sample, and panel B shows a side view.

\section{S2. rFRAP}

The rFRAP technique has previously been used to study transport in systems relevant in the material sciences and life sciences. ${ }^{4-6}$ Additionally, we have used rFRAP to measure diffusion coefficients in SOA proxies and SOA generated in an environmental chamber. ${ }^{7,8}$ In a rFRAP experiment, a rectangular area within a fluorescent thin film is photobleached with a laser scanning confocal microscope. Over time, bleached molecules diffuse outside of the bleached region and fluorescent molecules diffuse into the bleached region. From the rate of fluorescence recovery in the bleached area, diffusion coefficients are determined.

The rFRAP experiments were performed on a Zeiss Axio Observer laser scanning microscope (510MP). The pixel dwell time during scanning was $3.20-12.80 \mu \mathrm{s}$, with a scan time of $1.96-7.87 \mathrm{~s}$, depending on the level of fluorescence of the sample. The pinhole was set to $80 \mu \mathrm{m}$. A HeNe laser (543 $\mathrm{nm})$ was used to bleach an area of interest in the sample, as well as 
for imaging. Laser power for bleaching ranged from $243 \mu \mathrm{W}$ to $390 \mu \mathrm{W}$, depending on temperature and $a_{w}$ of the sample. Imaging after photobleaching was preformed with a laser power of $7.6 \mu \mathrm{W}$. Bleach depths (amount of fluorescence signal decrease due to photobleaching) ranged from around $20-50 \%$ with most experiments having a bleach depth of approximately $30 \%$. A bleach depth of $20-50 \%$ is within the range previously recommended. ${ }^{5}$

Heating of the sample by the laser during photobleaching was not expected to affect the diffusion measurements as previous measurements showed that diffusion coefficients were independent of the bleach area $^{8}$ and bleach laser intensity. ${ }^{7}$

Dimensions of the bleach area in the rFRAP experiments ranged from $4.88 \times 4.88 \mu \mathrm{m}$ to $19.89 \times 19.89 \mu \mathrm{m}$ depending on the recovery time. Smaller bleach areas were used when diffusion was slower to keep the recovery times $<1$ day.

Images recorded during rFRAP experiments were processed using ImageJ. The images were normalized by dividing all post-bleach images by an image taken immediately prior to bleaching. Each 512 x 512-pixel image obtained from an rFRAP experiment was averaged by downsizing to $128 \times 128$ pixels to reduce noise. Reported diffusion coefficients are the average of a minimum of 3 measurements taken at each temperature and $a_{w}$.

\section{S3. Data analysis}

Deschout et al. (2010) developed the following equation to describe the fluorescence intensity as a function of the $x$-position, $y$-position, and time $(t)$ after photobleaching a rectangular area with a confocal microscope: ${ }^{5}$

$$
\frac{F(x, y, t)}{F_{0}(x, y)}=1-\frac{K_{0}}{4}\left[\operatorname{erf}\left(\frac{x+\frac{l_{x}}{2}}{\sqrt{r^{2}+4 D t}}\right)-\operatorname{erf}\left(\frac{x-\frac{l_{x}}{2}}{\sqrt{r^{2}+4 D t}}\right)\right] \times\left[\operatorname{erf}\left(\frac{y+\frac{l_{y}}{2}}{\sqrt{r^{2}+4 D t}}\right)-\operatorname{erf}\left(\frac{y-\frac{l_{y}}{2}}{\sqrt{r^{2}+4 D t}}\right)\right]
$$


Where $F_{0}(x, y)$ is the fluorescence intensity prior to photobleaching, $K_{0}$ is a parameter associated with the fraction of molecules photobleached in the bleached area immediately after photobleaching, $l_{x}$ and $l_{y}$ are the dimensions of the bleach area ( $l_{x}=l_{y}$ in this work), $r$ is the resolution of the microscope, and $D$ is the diffusion coefficient (of R6G in this work). Eq. (S2) assumes uniform photobleaching in the z-coordinate (i.e. the axial direction). This assumption was shown to be appropriate by Deschout et al. (2010) if a low numerical aperture lens $(\leq 0.45)$ and thin film $(\leq 120 \mu \mathrm{m})$ was used. Following these guidelines, a numerical aperture of 0.30 and film thickness $<80 \mu \mathrm{m}$ was used in this work.

Eq. (S2) was fit to all the images recorded after photobleaching to determine a value for $r^{2}+4 D t$ corresponding to each image. To ensure adequate signal to noise, images were only used if the difference in fluorescence signal between the bleached and non-bleached region was greater than three times the noise in the images. Next, $r^{2}+4 D t$ for each image determined from the fitting process was plotted as a function of $t$, and a straight line was fit to the data (e.g. Fig. S2). From the slope of the line, the diffusion coefficient was determined (slope $=4 \times D$ ).

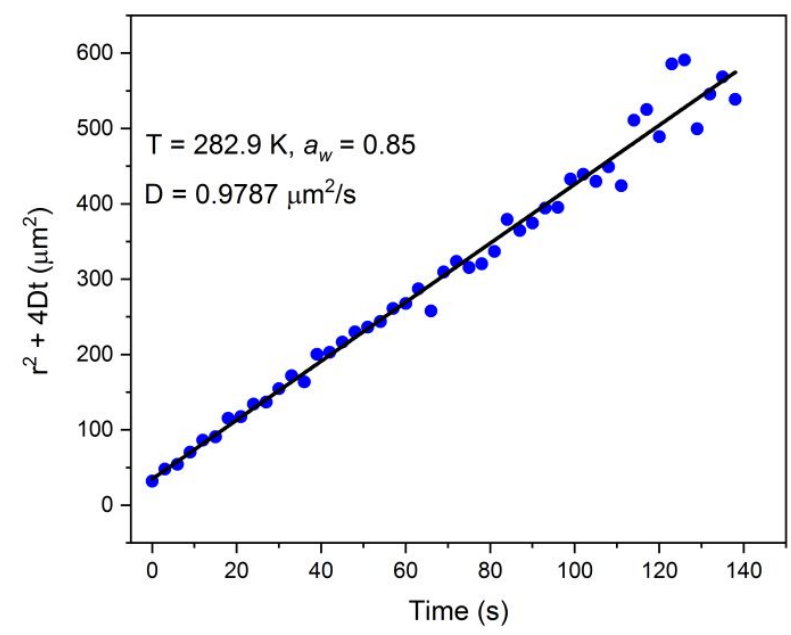

Figure S2. Plot of $r^{2}+4 D t$ against time for an rFRAP experiment at $a_{w}=0.85$ and $\mathrm{T}=282.9 \mathrm{~K}$, where $r$ is the lateral resolution of the microscope, $D$ is the diffusion coefficient and $t$ is time. The solid line is a linear fit to the data. 
The bleach width $\left(l_{x}, l_{y}\right)$ was allowed to be a free parameter in the fit of Eq. (S2) for the first 5 frames. The resulting bleach width was then used in the full analysis of the images.

Table S2. Diffusion coefficients determined in this study as a function of $a_{w}$ and temperature. Uncertainty in the $a_{w}$ values is from the uncertainty in the hygrometer. Uncertainty in temperature is from the $95 \%$ confidence interval of the temperature calibration (Section S4). The uncertainty in the diffusion coefficients represents the $95 \%$ confidence interval of at least 3 repeats.

\begin{tabular}{|c|c|c|}
\hline$a_{w}$ at $293 \mathrm{~K}$ & $\begin{array}{l}\text { Temperature } \\
(\mathrm{K})\end{array}$ & $\begin{array}{c}\text { Diffusion coefficient } \\
\left(\mathrm{m}^{2} / \mathrm{s}\right)\end{array}$ \\
\hline \multirow{4}{*}{$0.85 \pm 0.025$} & $282.9 \pm 0.5$ & $7.70 \times 10^{-13} \pm 1.91 \times 10^{-13}$ \\
\hline & $277.6 \pm 0.5$ & $5.24 \times 10^{-13} \pm 1.31 \times 10^{-13}$ \\
\hline & $272.3+0.6$ & $3.22 \times 10^{-13} \pm 0.46 \times 10^{-13}$ \\
\hline & $267.1 \pm 0.6$ & $2.26 \times 10^{-13} \pm 0.22 \times 10^{-13}$ \\
\hline \multirow{5}{*}{$0.75 \pm 0.025$} & $293.4+0.6$ & $2.05 \times 10^{-13} \pm 0.13 \times 10^{-13}$ \\
\hline & $288.1 \pm 0.5$ & $1.03 \times 10^{-13} \pm 0.09 \times 10^{-13}$ \\
\hline & $282.9 \pm 0.5$ & $5.40 \times 10^{-14} \pm 0.73 \times 10^{-14}$ \\
\hline & $272.3+0.6$ & $1.05 \times 10^{-14} \pm 0.07 \times 10^{-14}$ \\
\hline & $261.8 \pm 0.6$ & $1.58 \times 10^{-15} \pm 0.60 \times 10^{-15}$ \\
\hline \multirow{5}{*}{$0.59 \pm 0.025$} & $319.5 \pm 1.4$ & $6.31 \times 10^{-14} \pm 0.44 \times 10^{-14}$ \\
\hline & $314.3 \pm 1.2$ & $3.27 \times 10^{-14} \pm 0.17 \times 10^{-14}$ \\
\hline & $309.1 \pm 1.0$ & $1.59 \times 10^{-14} \pm 0.18 \times 10^{-14}$ \\
\hline & $303.8 \pm 0.8$ & $8.57 \times 10^{-15} \pm 0.98 \times 10^{-15}$ \\
\hline & $293.4+0.6$ & $1.34 \times 10^{-15} \pm 0.46 \times 10^{-15}$ \\
\hline \multirow{3}{*}{$0.43 \pm 0.025$} & $319.5 \pm 1.4$ & $1.08 \times 10^{-15} \pm 0.21 \times 10^{-15}$ \\
\hline & $314.3 \pm 1.2$ & $3.70 \times 10^{-16} \pm 1.14 \times 10^{-16}$ \\
\hline & $303.8 \pm 0.8$ & $6.13 \times 10^{-17} \pm 1.17 \times 10^{-17}$ \\
\hline
\end{tabular}




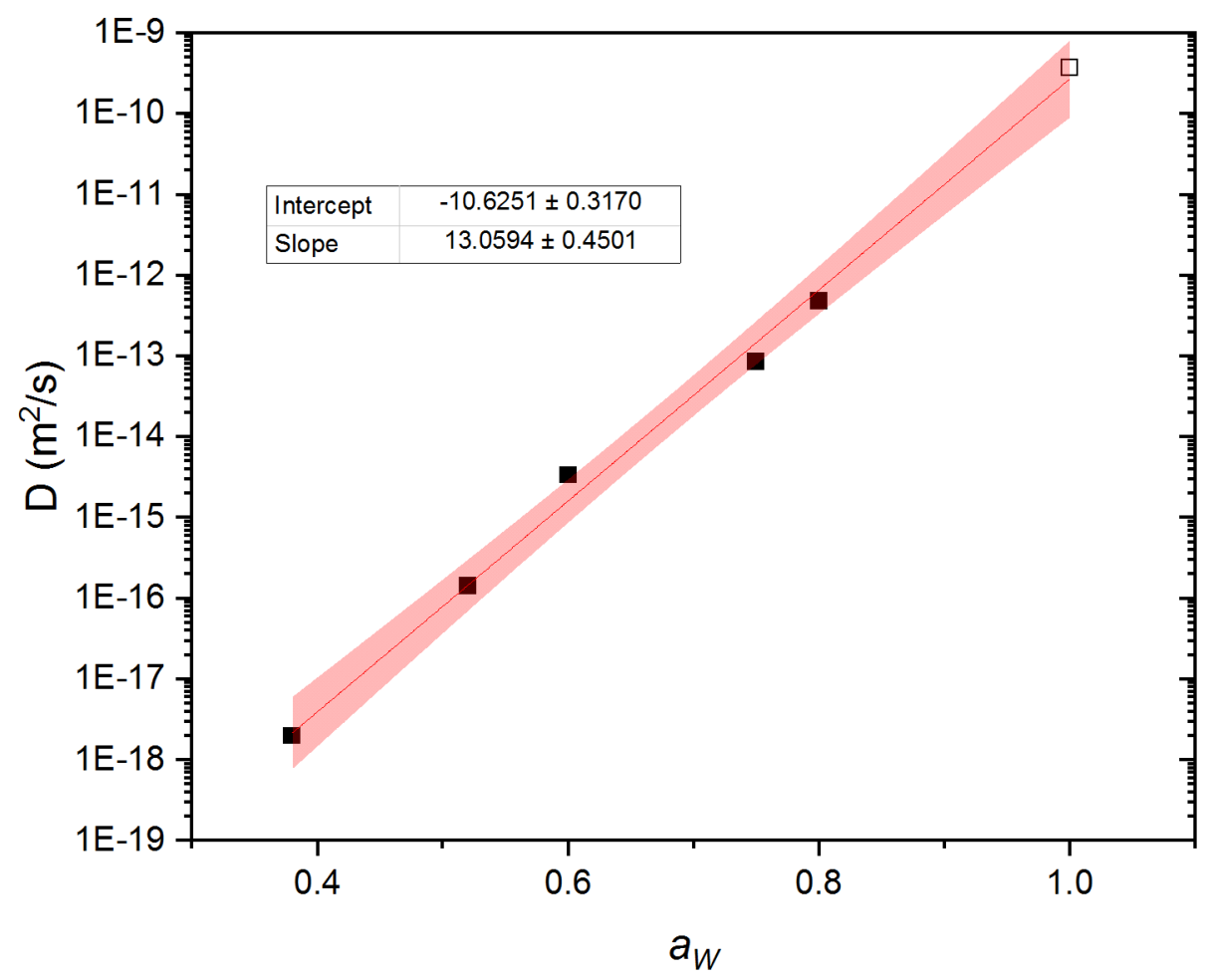

Figure S3. Plot of log diffusion vs $a_{w}$ for rhodamine-6G in sucrose-water mixtures at room temperature from Chenyakin et al. (2017). The black squares correspond to the measured values and the red line is a linear fit to the data with $95 \%$ confidence bands (red shaded area). The open symbol at $a_{w}=1$ is the diffusion of rhodamine-6G in pure water. ${ }^{9}$ Diffusion coefficients at $a_{w}$ values of $0.85,075,0.59$, and 0.43 were determined from the linear fit to the data. Uncertainties in the diffusion coefficients were calculated from the uncertainty in $a_{w}( \pm 0.025)$ from Chenyakin et al. (2017) and the $95 \%$ confidence bands. 


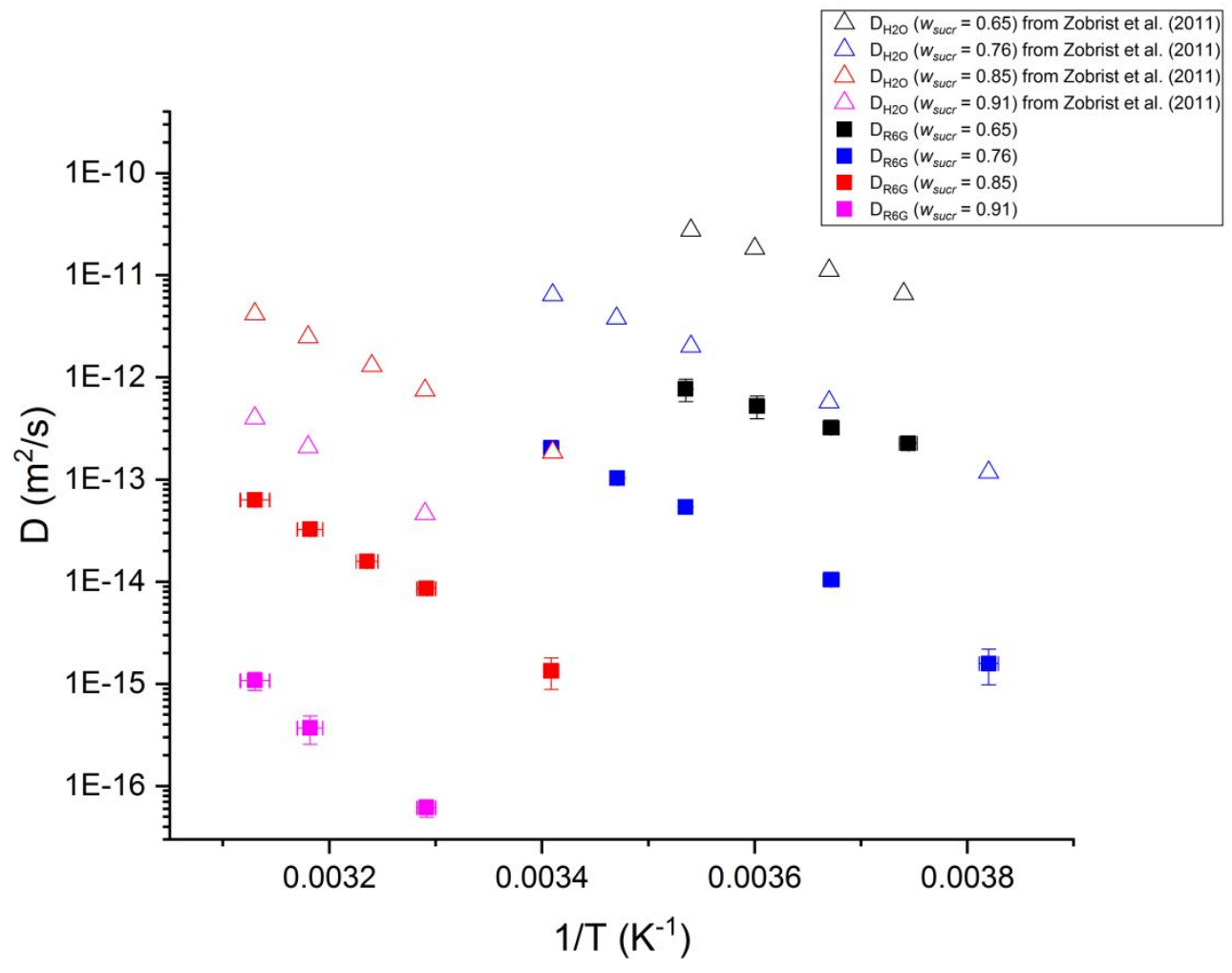

Figure S4. Diffusion coefficients of rhodamine-6G (closed squares) and water (open triangles) in sucrose-water as a function of temperature. Black, blue, red and magenta symbols correspond to $w_{\text {sucr }}$ values of $0.65,0.76,0.85$, and 0.91 respectively.

Uncertainty in diffusion for R6G is the $95 \%$ confidence interval of at least three trials. The uncertainty in temperature is from the temperature control stage calibration (Section S4). Diffusion coefficients for water were calculated from parameterization developed by Zobrist et al. (2011). ${ }^{10}$

\section{S4. Temperature control stage and calibration}

The sample in the rFRAP experiments was housed in a temperature control stage (HCS32lGi

INSTEC, Figure S5). The temperature of the stage was calibrated using known melting temperatures of chloroform $(209.85 \pm 0.6 \mathrm{~K})$, acetonitrile $(229.15 \pm 1 \mathrm{~K})$, dodecane $(263.60 \pm$ $0.02 \mathrm{~K})$, water $(273.15 \mathrm{~K})$, diphenyl ether $(300.02 \mathrm{~K})$ and 1-octadecanol $(331.15 \pm 2 \mathrm{~K}) .{ }^{11}$ 


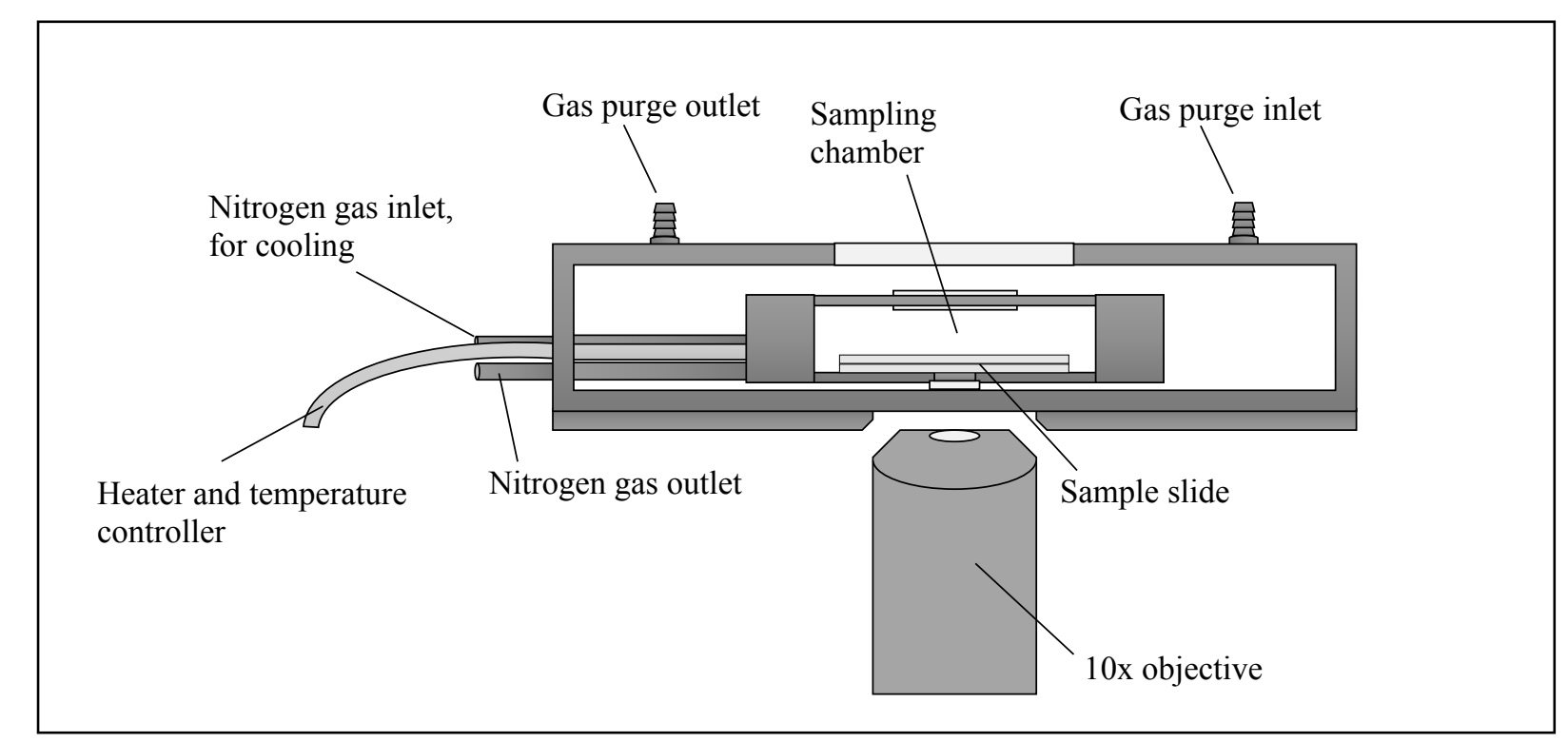

Figure S5. Schematic of the temperature control stage mounted above the 10x objective of the laser scanning microscope for rFRAP measurements.

Droplets (or crystals) of each substance were placed on glass slides. Materials which were liquid at room temperature were sealed between two glass slides with vacuum grease to prevent evaporation. Materials that were crystals at room temperature were placed on a glass slide but not sealed. When determining melting temperatures, the temperature was increased at a rate of $0.1 \mathrm{~K} / \mathrm{min}$. The temperature offset of the stage was determined by comparing the measured melting temperatures with the melting temperatures reported in the literature (Fig. S6). Each melting point was measured a minimum of four times, and the error bars represent the $95 \%$ confidence interval of the replicates. 


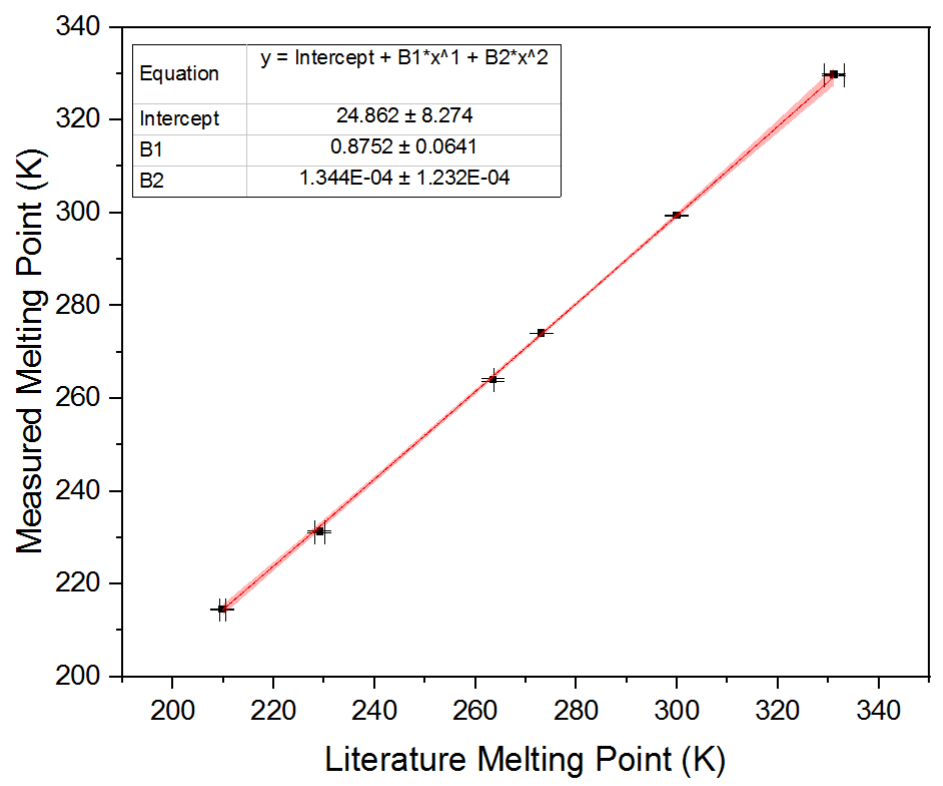

Figure S6. Calibration for the temperature control stage (HCS321Gi INSTEC). Measured melting temperatures of chloroform, acetonitrile, dodecane, water, diphenyl ether, and 1-octadecanol compared to their known melting points. ${ }^{11} \mathrm{~A} 2^{\text {nd }}$ order polynomial was fit to this data (red line) with $95 \%$ confidence bands (red shaded area). The $y$-error bars represent the $95 \%$ confidence interval of at least four measurements. 


\section{References:}

(1) Shiraiwa, M.; Ammann, M.; Koop, T.; Poschl, U. Gas Uptake and Chemical Aging of Semisolid Organic Aerosol Particles. Proc. Natl. Acad. Sci. 2011, 108 (27), 11003-11008. https://doi.org/10.1073/pnas.1103045108.

(2) Price, H. C.; Murray, B. J.; Mattsson, J.; O’Sullivan, D.; Wilson, T. W.; Baustian, K. J.; Benning, L. G. Quantifying Water Diffusion in High-Viscosity and Glassy Aqueous Solutions Using a Raman Isotope Tracer Method. Atmos. Chem. Phys. 2014, 14 (8), 38173830. https://doi.org/10.5194/acp-14-3817-2014.

(3) Greenspan, L. Humidity Fixed Points of Binary Saturated Aqueous Solutions. J. Res. Natl. Bur. Stand. Sect. A Phys. Chem. 1977, 81 A (1), 89. https://doi.org/10.6028/jres.081A.011.

(4) Deschout, H.; Raemdonck, K.; Demeester, J.; De Smedt, S. C.; Braeckmans, K. FRAP in Pharmaceutical Research: Practical Guidelines and Applications in Drug Delivery. Pharm. Res. 2014, 31 (2), 255-270. https://doi.org/10.1007/s11095-013-1146-9.

(5) Deschout, H.; Hagman, J.; Fransson, S.; Jonasson, J.; Rudemo, M.; Lorén, N.; Braeckmans, K.; van de Manakker, F.; Braeckmans, K.; Morabit, N.; et al. Straightforward FRAP for Quantitative Diffusion Measurements with a Laser Scanning Microscope. Opt. EXPRESS Biophys. J. J. Crank J. Microsc. Y. Pawitan, All Likelihood 2010, 19 (30), 2992-3001. https://doi.org/10.1364/OE.18.022886.

(6) Lorén, N.; Hagman, J.; Jonasson, J. K.; Deschout, H.; Bernin, D.; Cella-Zanacchi, F.; Diaspro, A.; McNally, J. G.; Ameloot, M.; Smisdom, N.; et al. Fluorescence Recovery after Photobleaching in Material and Life Sciences: Putting Theory into Practice. Q. Rev. Biophys. 2015, 48 (03), 323-387. https://doi.org/10.1017/S0033583515000013.

(7) Ullmann, D. A.; Hinks, M. L.; Maclean, A.; Butenhoff, C.; Grayson, J.; Barsanti, K.; Jimenez, J. L.; Nizkorodov, S. A.; Kamal, S.; Bertram, A. K. Viscosities, Diffusion Coefficients, and Mixing Times of Intrinsic Fluorescent Organic Molecules in Brown Limonene Secondary Organic Aerosol and Tests of the Stokes-Einstein Equation. Atmos. Chem. Phys. Discuss. 2019, 1-22. https://doi.org/10.5194/acp-2018-899.

(8) Chenyakin, Y.; Ullmann, A. D.; Evoy, E.; Renbaum-Wolff, L.; Kamal, S.; Bertram, K. A. Diffusion Coefficients of Organic Molecules in Sucrose-Water Solutions and Comparison with Stokes-Einstein Predictions. Atmos. Chem. Phys. 2017, 17 (3), 2423-2435. https://doi.org/10.5194/acp-17-2423-2017.

(9) Majer, G.; Melchior, J. P. Characterization of the Fluorescence Correlation Spectroscopy (FCS) Standard Rhodamine 6G and Calibration of Its Diffusion Coefficient in Aqueous Solutions. J. Chem. Phys. 2014, 140 (9). https://doi.org/10.1063/1.4867096.

(10) Zobrist, B.; Soonsin, V.; Luo, B. P.; Krieger, U. K.; Marcolli, C.; Peter, T.; Koop, T. Ultra-Slow Water Diffusion in Aqueous Sucrose Glasses. Phys. Chem. Chem. Phys. 2011, 13 (8), 3514. https://doi.org/10.1039/c0cp01273d.

(11) Rumble, R. J. CRC Handbook of Chemistry and Physics, 100th ed.; CRC Press/Taylor \& Francis: Boca Raton, FL, 2019. 